\title{
Termes éponymes en médecine et application pédagogique
}

\section{Sylvie Monin}

\section{(2) OpenEdition}

\section{Journals}

Édition électronique

URL : http://journals.openedition.org/asp/3527

DOI : 10.4000/asp.3527

ISSN : 2108-6354

\section{Éditeur}

Groupe d'étude et de recherche en anglais de spécialité

\section{Édition imprimée}

Date de publication : 1 décembre 1996

Pagination : 217-237

ISSN : 1246-8185

\section{Référence électronique}

Sylvie Monin, «Termes éponymes en médecine et application pédagogique », ASp [En ligne],

11-14 | 1996, mis en ligne le 08 mai 2013, consulté le 19 avril 2019. URL : http://

journals.openedition.org/asp/3527 ; DOI : 10.4000/asp.3527

Ce document a été généré automatiquement le 19 avril 2019.

Tous droits réservés 


\title{
Termes éponymes en médecine et application pédagogique
}

\author{
Sylvie Monin
}

Ce texte est dédié à Monsieur le Docteur Jean Cuche, chirurgien à l'Infirmerie protestante de Lyon

Seule l'expérimentation permet d'apporter la preuve des hypothèses avancées. Claude Bernard ( Médecine expérimentale 1865)

Il ne saurait exister de connaissances véritables en dehors de l'expérience des faits. Auguste Comte

\section{Introduction}

1 Cette étude porte sur les termes éponymes anglais et français en médecine, et suit celle présentée au colloque 1993 du GERAS sur la siglaison. En effet, il est intéressant de se pencher sur une autre forme de dénomination qui est elle aussi source de confusion et d'opacité sémantique.

2 Après avoir relevé les termes éponymes présents dans deux corpus médicaux rédigés, l'un en français, l'autre en anglais, et comportant des termes médicaux courants, nous avons tenté de répondre aux questions suivantes :

- Qu'est-ce qu'un éponyme?

- Quelles sont les limites de cette notion?

- L'emploi du terme éponyme en médecine est-il fréquent ? Pourquoi ?

- Est-ce que le médecin francophone utilise plus de termes éponymes que son collègue anglophone?

- L'emploi de termes éponymes en médecine reflète-t-il la chronologie de l'histoire de la médecine et de la santé ?

- Est-ce que le terme éponyme est dépourvu de toute connotation? 
3 Après qu'auront été définie la notion de «terme éponyme ", précisées les limites de cette notion, puis examiné le caractère connotatif de certains éponymes et la fréquence éponymique en terminologie médicale (à la fois en anglais et en français) et leurs différents types, on proposera une série d'exercices pédagogiques (qui n'ont pas été jusqu'ici envisagés dans les manuels de langue, notamment spécialisée). Ce travail se veut donc novateur, tout en s'appuyant sur des connaissances établies scientifiquement à partir d'une pratique liée à l'observation' ${ }^{1}$.

\section{Définition}

Selon le Grand Larousse Universel, l'adjectif éponyme - du grec epônumos, de epi, sur, et onoma, nom - rappelle l'existence du « héros » dans l'Antiquité, qui, « fondateur plus ou moins légendaire d'une cité, aurait donné son nom à cette cité ». Par exemple, Athena est la déesse éponyme d'Athènes.

C'est aussi le

magistrat annuel dont le nom servait d'indication chronologique et se donnait à

l'année... Un procédé de datation était en usage à Rome, au moyen des noms des deux consuls.

Quant au nom masculin éponyme, il sert à désigner un "héros » ou un «magistrat éponyme ». Par exemple, Romulus est éponyme de Rome (Larousse 1992 : 3831). Cet adjectif antique correspond au nom éponyme très vaguement défini dans le Nouveau Dictionnaire étymologique et historique: "celui qui donne son nom à quelque chose » ( Larousse 1971 : 400).

6 Selon le Grand Dictionnaire encyclopédique Larousse (1992: 3831), " un site éponyme à l'époque de la préhistoire est un site qui a donné son nom à une culture ou à un faciès industriel, et dont il est parfaitement représentatif ».

7 Apparaît alors le nom éponymie, qui sert à désigner « la fonction des magistrats éponymes et la durée de leur fonction » (id.).

8 En anglais, il existe aussi l'adverbe eponymously lui-même dérivé de l'adjectif eponymous comme cela est mentionné dans la définition suivante

The eponymous character in a book, film, or play is the character whose name forms its title: Hester, the book's eponymous heroine -eponymously, adverb ( Dictionary of Contemporary English 1995 : 456).

Du point de vue lexicologique, ce terme antique est défini de manière plus précise par $\mathrm{J}$. Tournier :

Les personnages qui donnent leur nom à un objet, ou une notion, sont dits éponymes. Par extension, on utilise aussi ce terme pour désigner les mots ainsi formés...

La métonymie onomastique est le changement fondé sur une association d'idées, d'un nom propre en nom commun, adjectif ou verbe. (1991:134)

10 Quant aux linguistes R.R.K. Hartmann et F. C. Stork, ils proposent cette définition fort limitée du terme « eponym » en anglais :

The name of a geographical location or institution based on the name of a person,

e.g., Leningrad or Washington. (Hartmann \& Stock : 1978)

11 Les définitions des spécialistes de médecine, bien que plus précises, restent encore insuffisantes comme le prouvent les définitions suivantes : 
Eponym - Gr. eponymos, named after - a name for anything - diseases, organs, functions, places - adapted from the name of a particular person. (Taber's Cyclopedic Medical Dictionary 1989)

Selon Jean Hamburger,

... on a pris l'habitude de désigner certains signes, certains syndromes, certaines maladies, certaines interventions chirurgicales, certains instruments, par le nom du médecin qui les décrivit. Celui-ci, pour reprendre un terme antique, devient l'éponyme de ce qu'il a trouvé. (1982).

Selon Steven Carter-Lovejoy (1988),

an eponym is a term derived from someone's name. Often an eponym gives credit to the person first describing a feature (Hodgkin's disease), but sometimes it makes a literary or mythic reference (Ulysse syndrome) or even immortalizes the unfortunate patient (Dusard syndrome).

12 À l'insuffisance des définitions, s'ajoute le problème du caractère flou, parfois, de la terminologie médicale; il s'agit, en particulier, du problème de la limite peu précise qui distingue entre eux les concepts de "syndrome» et "maladie», "symptômes » et «signe ». Par exemple, les savants francophones emploient indifféremment les termes «symptômes» (qui sont subjectifs) et «signes" (appelés habituellement «symptômes objectifs ») pour désigner les manifestations d'un état pathologique, alors que leurs collègues anglophones différencient bien nettement les termes «symptoms » (qui sont répartis en constatations - objective, subjective, cardinale, [car elle est liée à la constitution du corps du malade] et parfois constitutionnelle) et "signs » (qui est une constatation objective comme « avoir un gros cœur »).

En français, on ne fait pas toujours la distinction entre "maladie ", "syndrome », " affection » et " entité morbide » et on emploie ces termes comme des synonymes. De plus, certaines maladies, ayant des causes multiples, sont aussi appelées «syndromes». En anglais, le terme "syndrome " " sert à désigner un ensemble de symptômes qui se manifestent en même temps. En français, il s'agit d'un ensemble de signes, symptômes, lésions et de modifications fonctionnelles ou biochimiques formant une entité reconnaissable. En anglais, le terme « disease » est défini comme

a pathological condition of the body that presents a group of clinical signs and symptoms and laboratory findings. The concept of disease may include the condition of illness or suffering not necessarily arising from pathological changes in the body.

En français, le terme «maladie » sert à désigner toutes les manifestations pathologiques permettant de la reconnaître. En anglais, on différencie le terme "illness " ( which is highly individual and personal ») du terme " disease " (" which is usually tangible and may even be measured »). Il existe aussi des différences d'extension sémantique entre les termes « pathology» (ayant les deux sens suivants : 1) "Study of the nature and cause of disease involving changes in structure and function; 2) condition produced by disease ») et " pathologie " (qui désigne une partie de la médecine étudiant les maladies du point de vue clinique ou anatomique).

Le terme " affection » a un sens plus étendu en français qu'en anglais car il sert à couvrir les concepts « anomalie, dysfonction, lésion, maladie, syndrome, atteinte de l'organisme ou trouble des fonctions physiologiques ou psychiques» en médecine. Quant aux expressions "entité morbide " et "morbid entity ", elles réfèrent à un ensemble de manifestations pathologiques caractérisées par leur constance et leur groupement. 

anglais et en français - pour désigner la même pathologie. Par exemple, les anglophones utilisent l'expression éponyme « Addison's disease » correspondant à celle de « maladie de Royer » en français. l'occurrence, de simplifier les choses en utilisant, du côté anglophone, des synonymes descriptifs comme "adrenocortical hypofunction", "chronic hypoadrenocorticism» et, du côté francophone, l'emprunt du nom du médecin londonien, Thomas Addison (1793-1860), qui a donné la première description de cette insuffisance surrénale chronique due le plus souvent à la destruction complète ou partielle des deux glandes surrénales par des lésions tuberculeuses. Ses cinq manifestations sont l'anémie, l'asthénie, l'hypotension, des troubles gastriques et une coloration foncée de la peau.

\section{des raisons homonymiques :}

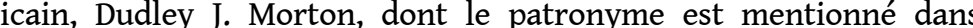
l'expression "Morton's disease », elle-même synonyme de "Morton's foot syndrome » désignant une affection caractérisée par un raccourcissement du premier métatarsien, ne doit pas être confondu avec le chirurgien américain, Thomas George Morton (1835-1903), dont le patronyme figure dans l'expression «Morton's neuralgia " qui sert à désigner la douleur située au niveau d'un métatarsien de l'orteil.

l'inexactitude de l'appellation éponymique initiale :

Selon Jean Hamburger, l'appellation «syndrome de Goodpasture » est inappropriée car elle associe une affection pulmonaire avec expectoration sanglante et une affection du rein «parce que ce n'est pas E. W. Goodpasture qui l'a décrite et que l'observation qu'il publia en 1919 ne ressemble que de loin à ce qu'on désigne aujourd'hui sous son nom » (1982: 14).

la confusion possible entre l'appellation éponymique et son dérivé :

21 Il ne faut pas confondre l'appellation éponymique "Addison's disease " (maladie liée à la destruction totale et bilatérale des glandes surrénales décrite par le médecin londonien, Thomas Addison, en 1855) avec le nom dérivé «addisonism» (nom propre Addison + suffixe - ism) qui sert uniquement à désigner la présence d'une pigmentation cutanée anormale avec une grande fatigabilité.

l'obsolescence de l'appellation éponymique :

L'appellation éponymique "Coats' disease» (rappelant la description réalisée par l'ophtalmologiste britannique, George Coats [1876-1915]) a beaucoup vieilli. À l'origine, elle servait à désigner de grandes taches blanches présentes dans les vaisseaux sanguins de la rétine. De nos jours, on utilise ce terme pour décrire au moins des troubles rétiniens bien distincts.

Parmi les limites notionnelles de l'appellation éponymique, figure aussi celle de « fauxéponymes». Par exemple, le terme "césarienne» n'est pas éponyme du personnage historique de César, qui, enfant, fut mis au monde par incision. C'est le chirurgien français, Ambroise Paré, considéré comme le père de la chirurgie moderne, qui fit entrer ce terme en français au seizième siècle. En effet, le terme "caesar " vient du verbe " caedere ", inciser en latin. En anglais, on utilise l'expression " cesarean section ", elle-même synonyme de « cesarotomy » et de « abdominal delivery ».

ASp, 11-14 | 2012 

Par exemple, le test APGAR (APGAR's test) ne vient pas du nom du pédiatre Virginia Apgar qui a mis au point cette méthode d'évaluation de l'état d'un nouveau-né, fondée sur la recherche des signes cliniques suivants

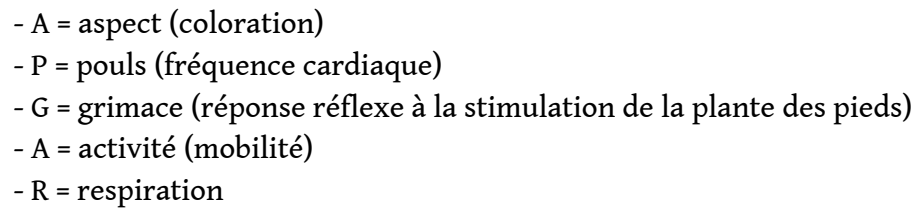

$\mathrm{Au}$ vu de toutes ces définitions et limites notionnelles, il est à noter que le terme éponyme n'est pas seulement un nom propre mais aussi un nom simple, un verbe ou un adjectif en dérivant. Alors, éponymer au sens strict, c'est emprunter le nom propre d'un individu ou celui d'un savant - découvreur ou inventeur -, et l'associer à un référent. En langue spécialisée, c'est un moyen commode d'accueillir un nouveau référent - découverte, instrument, théorie, maladie, syndrome - qui sera reconnu par les spécialistes-locuteurs concernés. Par exemple, l'expression éponyme "glandes de Bartholin " (Bartholin's glands) sert à désigner les glandes situées de chaque côté de la moitié postérieure de l'orifice vaginal, d'après la description réalisée par l'anatomiste danois Casper Bartholin (1655-1738). chimiste et biologiste français - Louis Pasteur (1822-1895) + suffixe -iser (-ize). Ce verbe sert à désigner l'opération de stérilisation d'une boisson fermentescible par échauffement sans ébullition, suivi d'un refroidissement brusque.

30 Il est à remarquer la règle orthographique suivante : le dérivé du nom propre ne prend pas de majuscule ni en français ni en anglais. De plus, une appellation éponyme peut comporter plusieurs noms propres placés à la suite, mais jamais plus de trois patronymes à la fois (elle est double ou triple). Par exemple,

31 - greffe d'ollier-Thiersch (Ollier-Thiersch graft) sert à appeler une greffe cutanée pratiquée avec des bandelettes dermo-épidermiques (d'après ollier, Léopold, chirurgien français [1822-1895]).

32 - coloration de May-Grünwald-Giemsa (en anglais, ne sont retenus que deux noms propres = May-Grünwald stain) sert à désigner un procédé de coloration des cellules sanguines à l'aide de deux colorants contenant de l'éosine et des dérivés de bleu de méthylène (d'après May, Richard, médecin allemand [1863-1937], Grünwald, Ludwig, otorhino-laryngologiste de Munich [né en 1863], et Giemsa, Gustave, médecin, chimiste et 
bactériologiste allemand [1867-1948]). Cette appellation éponyme est aussi synonyme de «méthode panoptique de Pappenheim» (d'après Pappenheim, Arthur [1870-1916], médecin allemand spécialisé en hématologie).

Les différents noms patronymiques sont reliés par des traits d'union. Cependant, il faut noter qu'il n'existe aucune distinction entre les noms patronymiques en composition et correspondant respectivement à plusieurs savants, et le patronyme d'un seul savant comportant plusieurs noms propres aussi en composition. Par exemple, l'appellation éponyme «albumosurie de Bence-Jones» (Bence-Jones proteinuria) désigne la présence dans les urines d'une protéine particulière - albumose ou protéine de Bence-Jones - et d'après un seul savant, un médecin britannique, Henry Bence-Jones (1813-1873).

Pour compléter cette définition du terme éponyme au sens strict, il peut être ajouté que ce type d'appellation est souvent facultatif, en raison de l'existence de synonymes. Par exemple,

- tache de Mariotte (Mariotte's spot) : cette expression désigne une région du champ visuel, d'après l'abbé Edmé Mariotte, physicien français (1620-1684). Elle a pour synonymes « tache aveugle », « point aveugle », « punctum coecum » et «blind point ».

36 - cavité de Retzius (Retzius' space) : cette expression sert à appeler un espace rempli de tissu cellulo-graisseux situé en avant de la vessie, d'après Retzius, Anders-Adolf, anatomiste suédois (1796-1860). Les synonymes sont "espace de Retzius", «espace prévésical », « retro-pubic space ».

37 - point de MacBurney (MacBurney's point) rappelle l'existence d'un point sensible à la palpation en cas d'appendicite, d'après Charles MacBurney, chirurgien de New York (1845-1914). Son synonyme est « point appendiculaire».

38 - kyste de Baker (Baker's cyst) sert à désigner une tuméfaction kystique constituée dans le creux poplité par l'inflammation de la bourse séreuse du muscle poplité (situé à la partie postérieure du genou), d'après Baker, William-Morrant, (1839-1896), chirurgien anglais. Son synonyme, "bursite poplité » ("popliteal bursitis») est surtout utilisé en Suisse Romande.

39 Au sens large, "éponymer » consiste à emprunter le nom propre d'un écrivain, d'un malade, d'un héros mythique ou romanesque, ou le nom d'un lieu (ou toponyme), d'un élément culturel ou encore celui d'un animal, et de l'associer à un référent (découverte, méthode, appareil, pathologie, etc.). Dans ce cas précis, on ne rend pas hommage à un membre appartenant à la communauté concernée. Par exemple :

40 a - le nom d'un animal: le terme "Rhésus» (Rhesus) existe d'après le nom du singe commun dans l'Inde - «Macacus Rhesus». Ce singe servi aux savants - Karl Landsteiner (biologiste américain d'origine autrichienne [1868-1943], aussi Prix Nobel de médecine en 1930) et son collègue Weiner en 1940, lors de leur expérience qui fut à l'origine de la découverte du facteur sanguin Rhesus (Rh).

41 b - le nom d'un lieu : L'expression « maladie de Lyme (« Lyme's disease », « Lyme arthritis ») sert à désigner une maladie endémique inflammatoire causée par un spirochète transmis par des tiques, notamment caractérisée par des arthrites de localisations diverses. Cette maladie fut identifiée pour la première fois en 1975, à Lyme, dans le sud-est de l'état du Connecticut qui se situe à douze miles à l'ouest de New London. Puis, elle fut repérée dans d'autres états américains, en Europe et en Australie. 

sert à désigner en médecine l'instrument servant à exciser les amygdales, d'après le médecin français, Joseph-Ignace Guillotin (1738-1814) qui, député, fit adopter par l'Assemblée législative un autre instrument aussi appelé de son nom patronymique en vue de remplacer les supplices par la décapitation. Elle fonctionna dès le 25 avril 1792 notamment à l'époque révolutionnaire, jusqu'à l'abolition de la peine de mort le 9 octobre 1981 pour les condamnés de droit commun.

$\mathrm{d}$ - le patronyme d'un écrivain : l'appellation « le signe de Musset » («Musset's sign ») sert à dénommer les secousses répétitives de la tête et du cou apparaissant en même temps que des contractions des ventricules du cœur dont a souffert le poète français Alfred de Musset (1810-1857). e - le patronyme d'un malade ou le nom de la catégorie professionnelle du malade : par exemple, - la maladie de Cowden (Cowden's disease) désigne une maladie systémique, à la fois cutanée et viscérale, d'après le nom patronymique du premier malade. Son synonyme est «syndrome des hamartomes multiples».

- maladie de Carrion (Carrion's disease) rappelle une maladie infectieuse fréquente au Pérou, au Chili, en Bolivie et en Colombie, d'après le malade Daniel-Alcides Carrion (1850-1885). Ses synonymes sont " anémie de Carrion », « bartonellose », « bartonellosis ».

47 - maladie de Christmas (Christmas disease) désigne l'hémophilie masculine. Ses synonymes sont « hémophilie B », " hemophilia B ».

48 L'appellation éponyme devient descriptive quand la profession ou l'activité du malade sont mises en avant.

49 - la maladie des légionnaires (legionnaires' disease) : cette expression sert à dénommer une pneumonie virale parfois accompagnée de troubles gastro-intestinaux et d'insuffisance rénale qui s'est produite pour la première fois en juillet 1976 parmi les membres de la Légion américaine descendus lors d'une réunion dans un hôtel de Philadelphie; ses synonymes sont "pneumonie des légionnaires", "fièvre de Pontiac", "pneumonie de Broad Street », « légionellose » et « legionellosis ».

50 - pianist's cramp = la crampe du ou des pianiste(s) (pour désigner les spasmes musculaires des doigts du pianiste) ;

51 - pigeon-breeder's disease = la maladie de l'éleveur de pigeons (pour désigner des frissons, fièvres et toux) ;

52 - shoemakers'cramp : la crampe des cordonniers (pour désigner les spasmes musculaires de la main et du bras des cordonniers);

53 - swimmer's ear : l'oreille du nageur (pour désigner un type d'otite externe).

$\mathrm{f}$ - le patronyme d'un personnage de roman ou de la littérature : l'appellation anglaise " oblomov syndrome » sert à désigner un refus de reprendre une vie normale après avoir été souffrant, d'après Llya Llych Oblomov, un personnage du roman Oblomov (publié en 1859) de l'écrivain russe, Ivan-Alek-Sandrovitch Gontcharov (1812-1891), qui est considéré comme le peintre de la décadence de la noblesse russe. Le terme éponyme «bovarysme » ( bovarysm) est un exemple de dérivation suffixale. Le patronyme "Bovary " (d'après le nom de l'héroïne du roman de Gustave Flaubert, écrivain français 1821-1881, Madame Bovary (Emma) qui est un personnage frappé d'idéalisme romantique [publié en 1857] + le suffixe -ysme [-isme], suffixe qui sert à désigner une condition ou une théorie, un 
principe ou une méthode). Il sert à dénommer un comportement qui consiste à fuir dans le rêve l'insatisfaction éprouvée dans la vie.

g - le patronyme d'un individu particulier: L'appellation éponyme "Sutton's law» (d'après le nom du braqueur américain de banques "Willie Sutton») repose sur le concept suivant : « un braqueur de banques se rend où il y a de l'argent ». En médecine, ce concept indique que «l'on devrait repérer les maladies où elles ont le plus de chances de s'y trouver ». Par exemple, la malaria se trouve dans les régions tropicales.

$\mathrm{h}$ - le nom d'un héros légendaire, mythologique ou biblique: le terme éponyme " morphine » ("morphine »), d'après Morphée, le dieu grec des songes, fils de la Nuit et du Sommeil, réfère à un alcaloïde présent dans l'opium découvert en 1804 par les savants Seguin, Courtois et Derosne. C'est aussi un narcotique et un analgésique très puissant. L'expression « Pomme d'Adam » (Adam's apple, elle-même synonyme de " Ponum Adami » et de «Prominentia laryngea »), d'après Adam qui a désobéi à son créateur en croquant la pomme tendue par son épouse, Éve. Selon certains chercheurs, il s'agit d'un abricot ou d'une figue au lieu d'une pomme. Ils furent donc chassés de leur paradis terrestre par la colère divine. Il s'agit du « péché originel » mentionné dans la bible ; cette expression sert à dénommer la saillie placée à la partie antérieure du cou, formée par le cartilage thyroïde. L'expression "tendon d'Achille» ("tendo calcaneus», "Achilles tendon »), d'après Achille, héros grec et roi des Myrmidons, qui fut touché mortellement au talon par une flèche empoisonnée; ce personnage du poème épique L'Iliade n'était vulnérable qu'au talon. Cette expression sert à appeler un volumineux tendon terminal du muscle triceps de la jambe qui s'insère sur la face postérieure du calcanéum.

i - le nom d'une population : l'expression éponyme «tablier des Hottentotes", (Hottentot bustle (apron); synonyme $=$ velamen vulvea), d'après Hottentote, la population du sudouest de l'Afrique, vivant notamment en Namibie, sert à désigner une malformation (ou "ventre en besace 1») chez les femmes hottentotes des petites lèvres qui, développées, peuvent atteindre jusqu'à 18 centimètres.

$\mathrm{j}$ - le nom de bienfaiteurs, philanthropes ou d'humanistes: l'expression anglaise " Nightingale Pledge » (« l'engagement /la promesse/le serment » de Nightingale (selon le contexte dans lequel est utilisé le terme «pledge»), d'après Florence Nightingale, philanthrope britannique (1820-1910) (qui, après avoir été infirmière auprès des soldats blessés de la guerre de Crimée entre 1854 et 1856, participa à la création des premières écoles d'infirmières ouvertes à Londres, en 1848 à l'hôpital St Jean l'Évangéliste et en 1860 à l'hôpital St Thomas) Sert à dénommer un «serment » moral et éthique concernant la pratique des soins infirmiers. En raison des découvertes médicales enregistrées au cours du dix-neuvième siècle, l'auxiliaire du médecin se devait d'acquérir une formation professionnelle et scolaire. Des médecins « hygiénistes » et des « humanistes » œuvrèrent donc pour créer des formations en soins infirmiers. L'expression anglaise " Nobel prize » (le prix Nobel) rappelle qu'un industriel et chimiste suédois - Alfred Nobel (1833-1896) fonda, par son testament, des récompenses annuelles au profit de savants ou d'auteurs dès 1901 dans les domaines suivants : physique, physiologie et médecine, littérature et paix. Les travaux en sciences économiques ont été récompensés dès $1969^{2}$.

De plus, certains termes éponymes ne sont pas dénués de connotations culturelles, religieuses, «nationales », désuètes et de « notoriété ».

Illustrons ces connotations par des exemples : 
61 a - des connotations culturelles: l'expression «syndrome de Pheidippides " ( Pheidippides syndrome), d'après Pheidippides, le célèbre coureur de marathon en 490 avant J.-C. est employée pour désigner des accidents mortels (le plus souvent des infarctus du myocarde) survenus pendant ou après une course de fond.

b - des connotations religieuses: l'expression «la pomme d'Adam» (Adam's apple) connote le Péché originel et sert à désigner une saillie médiane du cou, à la face antérieure du cartilage thyroïde qui est plus marquée chez l'homme que chez la femme.

63 c - des connotations nationales : le terme éponyme «Kwashiorkor » est ghanéen et emprunté en anglais et en français pour désigner un ensemble de troubles graves découlant d'une carence nutritionnelle (surtout en protéines) apparaissant chez le jeune enfant après le sevrage. Au Ghana, chez les enfants, c'est la maladie dont souffre l'aîné quand le cadet vient au monde.

$\mathrm{d}$ - des connotations « désuètes » :

64 - L'expression «Antigène Australie » (Australia antigen) est remplacée par « HBs Ag » (antigène de surface), « hépatite à antigène Australie » (hepatitis B) pour désigner une protéine sérique constituant l'antigène caractéristique de l'hépatite $B$ ou hépatite d'inoculation. On utilise encore le toponyme Australie car cet antigène a été découvert dans le sérum d'un aborigène australien.

65 - L'expression « œufs de Naboth » (nabothian cysts) sert à désigner de petits kystes formés par les glandes mucipares obstruées de la muqueuse du col utérin. Martin Naboth (anatomiste et médecin allemand, professeur à Leipzig, 1675-1721) prit pour des œufs ces kystes décrits comme étant « tombés de la cavité utérine et greffés sur le col de l'utérus ».

66 - L'expression « danse de Saint Guy » est le synonyme désuet de « maladie de Saint Guy » ou de "chorée de Sydenham " (Saint Vitus' dance, Sydenham's chorea) (d'après Thomas Sydenham, médecin de Londres, 1624-1689). Elle sert à nommer une affection nerveuse de la deuxième enfance, d'étiologie souvent rhumatismale.

67 e - des connotations de «notoriété» notamment scientifique : L'expression « un parkinson" est utilisée en langage clinique courant pour désigner "une paralysie agitante ». Elle connote la notoriété du découvreur James Parkinson (médecin anglais, 1755-1824), de l'affection neurologique due à des lésions dégénératives du corps strié et du locus niger, caractérisée cliniquement par un tremblement lent qui est encore appelée "maladie de Parkinson" (Parkinson's disease). Le nom "pasteurisation" ( pasteurization) vient du nom du chimiste et bactériologiste français, Louis Pasteur, qui a inventé le procédé de conservation temporaire de certains produits alimentaires pour éliminer des germes d'un seul chauffage à moins de $100^{\circ} \mathrm{C}$.

\section{La fréquence des termes éponymes en médecine}

L'enquête sur la fréquence des termes éponymes en médecine a été réalisée à partir d'un premier corpus médical en français (avec traduction en anglais des termes éponymes repérés) d'environ 11750 termes-occurrences recensés dans le Dictionnaire médical (qui reprend en abrégé le Dictionnaire français de médecine et de biologie en quatre volumes, par L. Manuila, A. Manuila et M. Nicoulin, 1992, 5 édition complétée), et d'un second corpus médical en anglais, plus important, d'environ 60000 termes occurrences (pour vérifier les tendances notées lors de l'examen du premier corpus) repérés dans Taber's encyclopedic 
medical dictionary. Ces corpus ont été choisis car ils comportent l'essentiel des termes actuels utilisés par les personnels médicaux et paramédicaux lors de leur communication spécialisée et quotidienne. De plus, ils ont l'avantage d'être récents - bien que l'une des limites méthodologiques résiderait dans la rapidité des progrès médicaux et nationaux. Avant de présenter les résultats de cette enquête, il faut mentionner qu'un terme éponyme a été considéré comme une seule occurrence, même s'il comporte plusieurs patronymes juxtaposés où est parfois le produit d'une dérivation suffixale.

Tableau 1. Distribution des termes éponymes-occurrences repérés à partir du premier corpus

\begin{tabular}{|l|l|l|l|}
\hline $\begin{array}{l}\text { nb termes éponymes- } \\
\text { occurrences }\end{array}$ & $\begin{array}{l}\text { Gn } \\
\text { GB }\end{array}$ & pour désigner \\
\hline 242 cas & 113 & 129 & anatomie \\
\hline 180 & 86 & 94 & maladie, syndrome, signe, opération \\
\hline 135 & 65 & 70 & test, calculs, formule, loi, réaction, procédé \\
\hline 61 & 30 & 31 & sonde, appareil, méthode \\
\hline 34 & 15 & 19 & incidence radiographique \\
\hline 15 & 5 & 5 & théorie, conception, système \\
\hline 44 & 22 & 22 & unité de mesure \\
\hline 50 & 25 & 25 & vaccin, virus, bacille, cellule, corps, corpuscule \\
\hline 131 & 58 & 73 & $\begin{array}{l}\text { héros mythiques ou légendaires, malades, faits } \\
\text { historiques ou culturels toponymes etc. }\end{array}$ \\
\hline Total : 887 & & & \\
\hline
\end{tabular}

Tableau 2. Distribution des termes éponymes-occurrences présents dans le second corpus

\begin{tabular}{|l|l|}
\hline $\begin{array}{l}\text { nombre de termes éponymes-occurrences en } \\
\text { anglais }\end{array}$ & pour désigner \\
\hline 483 cas & maladie, syndrome, signe \\
\hline 359 & anatomie \\
\hline 176 & test, calculs, etc. \\
\hline 74 & appareils, sondes \\
\hline 4 & incidence radiographique \\
\hline 40 & théorie \\
\hline
\end{tabular}




\begin{tabular}{|l|l|}
\hline 23 & unité de mesure \\
\hline 104 & vaccin, virus, bacille \\
\hline 30 & opération \\
\hline 8 & référence biblique \\
\hline 5 & malade \\
\hline 3 & récompense \\
\hline 16 & mythologie : Antiquité \\
\hline 26 & poète, personnage de roman, profession ou \\
\hline 4 & activité \\
\hline 69 & faits historiques ou culturels \\
\hline Total : 1424 cas & toponymes, dates, bâtiments \\
\hline
\end{tabular}

Les résultats de ces deux tableaux montrent que le nombre de termes éponymes est plutôt faible car il est inférieur à $10 \%$ dans les deux langues. Ce nombre est moindre en anglais, alors même que le corpus anglais est plus important. Les spécialistes francophones et anglophones semblent limiter leur emploi des termes éponymes pour éviter l'opacité et la confusion sémantiques. Le léger écart enregistré entre le nombre de termes éponymes français $(3.98 \%)$ et celui des termes éponymes anglais (3.98\% dans le tableau $\mathrm{n}^{\circ} 1$ et $2.37 \%$ dans le tableau $\mathrm{n}^{\circ} 2$ ) s'explique par l'emploi, prisé, des synonymes décrivant le référent en anglais au lieu de l'emprunt des patronymes des savants. Par exemple, l'expression éponyme «le canal de Wharton ", d'après le médecin anatomiste britannique, Thomas Wharton (1614-1673), est équivalente à l'expression descriptive « submandibular duct » en anglais, le canal excréteur de chacune des deux glandes salivaires sous-maxillaires. De fait, les spécialistes anglophones et francophones se servent principalement des termes éponymes pour dénommer des maladies, syndromes et des découvertes en anatomie.

Tableau 3. Distribution de l'appellation éponyme selon l'époque, le pays et les fonctions du savant

\begin{tabular}{|l|l|l|l|}
\hline Référent & Corpus & français & \\
\hline & siècle & pays & fonctions \\
\hline anatomie & $19-20^{\mathrm{e}}$ & $\begin{array}{l}\text { Allemagne } \\
\text { Grande Bretagne } \\
\text { France }\end{array}$ & $\begin{array}{l}\text { chirurgiens } \\
\text { anatomistes }\end{array}$ \\
\hline
\end{tabular}




\begin{tabular}{|c|c|c|c|}
\hline maladie, signe syndrome pathologie & $19-20^{\mathrm{e}}$ & $\begin{array}{l}\text { Allemagne } \\
\text { France } \\
\text { USA }\end{array}$ & médecins \\
\hline $\begin{array}{l}\text { test, réaction } \\
\text { épreuve } \\
\text { méthode }\end{array}$ & $19-20^{\mathrm{e}}$ & $\begin{array}{l}\text { Allemagne } \\
\text { USA }\end{array}$ & $\begin{array}{l}\text { médecins } \\
\text { bactériologistes }\end{array}$ \\
\hline appareil & $20^{\mathrm{e}}$ & $\begin{array}{l}\text { USA } \\
\text { France } \\
\text { Allemagne }\end{array}$ & $\begin{array}{l}\text { chirurgiens } \\
\text { médecins }\end{array}$ \\
\hline $\begin{array}{l}\text { théorie } \\
\text { conception } \\
\text { système } \\
\text { classification }\end{array}$ & $19-20^{\mathrm{e}}$ & Europe & scientifiques \\
\hline unité de mesure & $18-20^{\mathrm{e}}$ & $\begin{array}{l}\text { Allemagne } \\
\text { Angleterre }\end{array}$ & physicien \\
\hline $\begin{array}{l}\text { bacille } \\
\text { vaccin }\end{array}$ & $19-20^{\mathrm{e}}$ & $\begin{array}{l}\text { Allemagne } \\
\text { France } \\
\text { Italie, USA }\end{array}$ & bactériologistes \\
\hline & Corpus & anglais & \\
\hline anatomie & $19-20^{\mathrm{e}}$ & $\begin{array}{l}\text { Allemagne } \\
\text { France } \\
\text { Grande-Bretagne }\end{array}$ & anatomistes \\
\hline maladie, etc. & $19-20^{\mathrm{e}}$ & $\begin{array}{l}\text { Allemagne } \\
\text { France, USA } \\
\text { Grande-Bretagne }\end{array}$ & médecins \\
\hline test, etc. & $19-20^{\mathrm{e}}$ & $\begin{array}{l}\text { USA, Allemagne } \\
\text { France } \\
\text { Grande-Bretagne }\end{array}$ & médecins \\
\hline appareil & $19-20^{\mathrm{e}}$ & USA & chirurgiens \\
\hline théorie etc. & $19-20^{\mathrm{e}}$ & Europe, USA & médecins \\
\hline unité de mesure & $19-20^{\mathrm{e}}$ & $\begin{array}{l}\text { France } \\
\text { Allemagne } \\
\text { Grande-Bretagne }\end{array}$ & physiciens \\
\hline
\end{tabular}




\begin{tabular}{|l|l|l|l|}
\hline bacille & $19-20^{\mathrm{e}}$ & Allemagne, France & $\begin{array}{l}\text { médecins } \\
\text { bactériologistes }\end{array}$ \\
\hline
\end{tabular}

70 Les termes éponymes utilisés par les spécialistes anglophones et francophones témoignent de façon unanime des principaux événements qui ont marqué l'histoire de la médecine et de la santé. Les spécialistes en exercice rencontrent essentiellement, lors de leurs différentes situations de communication, des patronymes de médecins et de chirurgiens, ayant principalement vécu aux dix-neuvième et vingtième siècles en Europe (où l'ère des traitements génétiques et des découvertes de la biologie moléculaire) marqués par la découverte des microbes, de l'électroradiologie, de la radioactivité (1896), du radium (1898) et des anesthésiques. Ce tableau $n^{\circ} 3$ révèle une seule exception: les patronymes de médecins ou de chirurgiens, surtout d'origine américaine, servent à désigner des appareils et des méthodes, découverts au vingtième siècle. En effet, ce siècle est caractérisé par l'affluence des technologies médicales sophistiquées reposant sur la micro-informatique, les ondes électro-magnétiques, l'électricité, le rayonnement et les isotopes, etc., la physique et la chimie. Quant aux unités de mesure, les spécialistes utilisent des dénominations réalisées à l'aide de patronymes portés par des physiciens ayant vécu entre les dix-huitième et vingtième siècles. En effet, des progrès réalisés en sciences physiques facilitèrent les progrès médicaux. Par exemple, on ouvrit en 1776 la toute première boutique d'électricité médicale à Paris.

\section{Les principaux emplois des termes éponymes}

71 Six emplois des termes éponymes ont été observés lors du dépouillement des deux corpus. Il s'agit de l'emploi des termes éponymes afin de

72 4.1. Combler une lacune linguistique (c'est-à-dire dénommer sur le champ un référent avant de lui donner d'autres appellations synonymes parfois trop descriptives, longues et peu commodes à employer dans une conversation). Par exemple, le nom "wirsungographie» ("wirsungography») sert à désigner la radiographie du canal de Wirsung ; Johann Georg Wirsung (1600-1643) est l'anatomiste bavarois qui a observé pour la première fois le canal pancréatique appelé « canal de Wirsung » (patronyme Wirsung + « $\mathrm{O}$ » voyelle de liaison + -graphie suffixe indiquant un enregistrement). En anglais, par exemple, on utilise l'expression "parotid duct» alors qu'en français, on trouve une expression éponyme "canal de Stenon », d'après Stenon (Steno, Stenonius ou Stensen), Niels, anatomiste et théologien danois (1638-1686), pour appeler le canal excréteur de la glande parotide qui s'ouvre dans la bouche.

73 4.2. Rendre hommage au savant qui a fait des découvertes scientifiques. L'appellation scientifique «bacille de Koch» («Bacillus tuberculosis») sert à dénommer l'agent spécifique de la tuberculose qui a été découvert en 1882 par Robert Koch (1843-1910), bactériologiste et médecin hygiéniste allemand, qui a reçu le Prix Nobel de médecine en 1905.

74 4.3. Désigner l'une des composantes d'un même référent de base ou générique. Le terme " vaccin », selon les maladies prévenues, adopte, par exemple, les composantes suivantes:

75 - «vaccin de Pasteur» («rabies vaccine ») : c'est un vaccin antirabique préparé à partir d'un virus vivant atténué (d'après Louis Pasteur, chimiste et bactériologiste, 1822-1895). 
76 - « vaccin de Sabin-Koprowski » («Sabin's oral poliomyelitis vaccine ») qui est un type de vaccin antipoliomyélitique, (d'après Albert-Bruce Sabin, virologiste américain [New York] né en 1906, et Hilary Koprowski, virologiste américain né en 1916).

77 - « vaccin de Salk» (Salk vaccine) qui est un type de vaccin antipoliomyélitique créé en 1954 (d'après Jonas Edward Salk, un virologiste américain né à Pittsburg en 1914).

78 - «vaccin de Fermi » (Fermi's vaccine), qui est un type de vaccin antirabique préparé à partir d'un virus atténué et inactivé par le phénol (d'après Claudio Fermi, hygiéniste italien, 1862-1952).

79 - « vaccin B.C.G. » : les initiales B.C.G. désignent le bacille bilié de Calmette-Guérin (« B.C.G. vaccine »). C'est un vaccin antituberculeux comportant des bacilles tuberculeux bovins vivants qui ont perdu leur virulence après de nombreux passages sur milieu bilié (d'après Albert Calmette, bactériologiste français, qui fut sous-directeur de l'Institut Pasteur de Paris [1863-1933], et Camille Guérin, sérologiste de Paris, [1872-1961]).

80 4.4. Éviter la longueur d'une paraphrase descriptive ou l'emploi de termes savants difficiles à prononcer ou des appellations nationales. Par exemple, l'appellation «l'articulation tarso-métatarsienne» («tarsometatarsal joint») est synonyme de « l'articulation de Lisfranc ». Jacques Lisfranc (1790-1847) a été le chirurgien parisien qui a observé l'ensemble des articulations qui, au pied, rassemblent l'arcade tarsienne à la base des cinq métatarsiens. L'expression « bursite poplitée » est surtout utilisée en Suisse romande. En France, on utilise l'appellation éponyme « kyste de Baker » (« Baker's cyst »), d'après William Morrant Baker, chirurgien britannique (1839-1896), pour dénommer un kyste synovial. L'expression "oligophrénie phénylpyruvique» ("phenylpyruvic acid oligophrenia») est synonyme de "» maladie de Fölling", d'après le patronyme du physiologiste norvégien (né en 1888) Ivar Asbjörn Fölling. Il a décrit une affection héréditaire récessive, due à un trouble enzymatique gênant la dégradation d'un acide aminé, la phénylalanine.

81 4.5. Changer une dénomination devenue obsolète, si le référent concerné a fait l'objet de nouvelles recherches. L'expression «cul-de-sac vésico-rectal » ("Douglas' pouch ») peut être remplacée par l'appellation éponyme «cul-de-sac de Douglas » (d'après l'anatomiste écossais James Douglas [1675-1742], afin de désigner le point le plus bas de la cavité péritonéale).

82 4.6. Employer un toponyme pour mieux se rappeler le lieu de découverte d'une maladie (voir Tableau 4).

Tableau 4

\begin{tabular}{|c|c|c|}
\hline français & anglais & lieux \\
\hline $\begin{array}{l}\text { siamois } \\
\text { (jumeaux rattachés l'un à l'autre par deux } \\
\text { parties homologues de leurs corps) }\end{array}$ & $\begin{array}{l}\text { Siamese } \\
\text { twins }\end{array}$ & $\begin{array}{l}\text { Thailand } \\
\text { (anciennement Siam, état de l'Asie du } \\
\text { sud-est) }\end{array}$ \\
\hline eau de Javel & Javel water & Javel (partie de Paris) \\
\hline
\end{tabular}




\begin{tabular}{|c|c|c|}
\hline fièvre de Malte & Malta fever & $\begin{array}{l}\text { Malte (île d'un archipel de la } \\
\text { Méditerranée entre la Sicile et } \\
\text { l'Afrique) }\end{array}$ \\
\hline $\begin{array}{l}\text { vert-de-gris } \\
\text { ancien français } \\
\text { =vert de Grèce } \\
\text { hydrocarbonate de cuivre) }\end{array}$ & $\begin{array}{l}\text { verdigris } \\
\text { (Green of } \\
\text { Greece) }\end{array}$ & Grèce, état du sud-est de l'Europe \\
\hline sodomie & sodomy & $\begin{array}{l}\text { Sodome, } \\
\text { ancienne ville cananéenne } \\
\text { (aujourd'hui Sedom) }\end{array}$ \\
\hline
\end{tabular}

\section{Le terme éponyme en médecine et son application pédagogique}

En général, l'utilisation des termes éponymes n'est pas encouragée par les professeurs de médecine en raison de leur caractère confus et vague. De plus, leur trait culturel n'est pas toujours fiable. Par exemple, l'expression éponyme "pentalogie de Fallot» sert à désigner une cardiopathie congénitale ayant des malformations anatomiques d'une tétrade de Fallot, auxquelles s'ajoute l'anomalie suivante: la communication interauriculaire. Elle est attribuée au médecin français, Étienne Fallot (1850-1911) même s'il ne l'a pas décrite. L'appellation " gélatine de Chine » (en anglais = agar), synonyme des appellations " agar ", « agar-agar » et " gélose », comporte le toponyme "Chine » qui n'a aucun lien direct avec le lieu de fabrication de cette substance mucilagineuse qui est le Japon ${ }^{3}$.

Quant au professeur de langue spécialisée, il aura surtout pour objectif de répondre aux besoins spécifiques des professionnels de la santé, soit :

- comprendre et mémoriser les termes éponymes fondamentaux afin de se constituer « un bagage personnel » et de faciliter la communication professionnelle ;

- se familiariser à la dérivation suffixale ;

- se construire un savoir sur la chronologie de l'histoire de la médecine et de la santé afin de développer par la suite des capacités d'expression à la fois orale et écrite ;

- se familiariser avec les ambiguités soulevées par certains termes éponymes.

À titre d'illustration, quelques exemples d'exercices sont proposés en annexe afin de faciliter une approche communicative de l'enseignement des langues spécialisées, qui doit tenir compte sur le vif des différents comportements psychologiques et cognitifs des apprenants.

\section{Conclusion}

Cette étude a mis en valeur non seulement le rôle référentiel des patronymes utilisés en terminologie médicale mais aussi celui de «mémoire» de la discipline. Certains termes éponymes ont des connotations culturelles, religieuses, nationales, etc. Elle a aussi 
montré que l'emploi du nom propre, patronyme ou toponyme, est un acte de référence dénominative directe qui n'est pas exempt d'ambiguïtés sémantiques ou d'erreurs lorsqu'on a attribué initialement au référent (maladie, instrument, théorie, etc.) le nom de famille d'un savant qui n'est pas toujours le véritable découvreur. De plus, le caractère dénominatif $\mathrm{du}$ terme éponyme parfois devenu obsolète a parfois entrâné la multiplication des synonymes beaucoup plus descriptifs et neutres. C'est ce qui explique la faible fréquence des éponymes au sein des deux corpus dépouillés. À l'échelle internationale, les spécialistes anglophones et francophones de la médecine limitent leur emploi des termes éponymes même s'il subsiste lors des communications professionnelles quotidiennes. Cependant, l'emploi des termes éponymes reflète la chronologie de l'histoire de la discipline et fait appel, dans certains cas, à la dérivation suffixale. Si l'utilisation des termes éponymes n'est pas toujours encouragée par le professeur de la spécialité en raison de leur caractère trop vague et confus, il appartient, cependant, au professeur de langue spécialisée de donner des exercices facilitant une meilleure mémorisation des termes éponymes utiles par les personnels médicaux et paramédicaux, qui peuvent ainsi se constituer un bagage culturel personnel, en se familiarisant avec la dérivation suffixale ainsi qu'avec les ambiguïtés sémantiques découlant de l'emploi de ces termes.

$87 \mathrm{Au}$ demeurant, et malgré les inconvénients qu'elles comportent, les appellations éponymes restent encore employées de façon ordinaire car, in fine, elles confèrent un peu "d'humanité » à une terminologie qui tend de plus en plus à se déshumaniser, dans le sens de l'oubli progressif des patronymes des individus qui ont apporté leur pierre à l'édifice de la science, considérée à l'échelle internationale.

\section{BIBLIOGRAPHIE}

\section{Relatives au corpus}

Dictionnaire Encyclopédique Larousse. 1992. Paris : Larousse.

Dictionary of Contemporary English. 1995. Harlow : Longman.

Hartmann, R.R.K. \& F.C. Stork. 1978. Dictionary of Language and Linguistics. Londres : Applied Science Publishers Ltd.

Manuila, L., A. Manuila et M. Nicoulin. 1992. Dictionnaire médical. Collection « Abrégés ». Paris :

Masson.

Nouveau Dictionnaire Étymologique et Historique. 1971. Paris : Larousse.

TABER's Cyclopedic Medical Dictionary. 1989. 16th ed. Philadelphia : Clayton L. Thomas, \& F.A. Davis Co.

Tournier, J. 1991. Structures lexicales de l'anglais. Paris : Nathan.

Relatives aux termes éponymes 
Carter-Lovejoy, S. 1988. « Dictionary of medical eponyms ». Medical Reference Services Quarterly, 7/4, Haworth Press, 93-95.

Durham, R.H. 1960. Encyclopedia of Medical Syndromes. New York : Hoeber.

Firkin, B. G. \& J.A. Whitworth. 1987. Dictionary of Medical Eponyms. Parkridge-Parthenon Publishing Group.

Jablonski, S. 1969. Illustrated Dictionary of Eponic Syndromes and Diseases and their Synonyms.

Philadelphie : Saunders.

Jablonski, S. 1991. Dictionary of Syndromes and Eponic Diseases. Melbourne : Krieger.

Magalini, S. \& E. Scascia. 1981. Dictionary of Medical Syndromes. 2nd ed, Philadelphie : Lippincott.

Rodin, A.E. \& J. Dey. 1988. Encyclopedia of Medical Eponyms derived from Literacy Characters.

Melbourne : Krieger.

Roeder, C.S. 1988. « Locating information on medical syndromes ». Medical Reference Services Quarterly 17/2, Haworth Press, 23-30.

\section{Relatives à la science médicale}

Belbenoitavich, P.M. 1992. La Recherche documentaire et bibliographique en médecine et en pharmacie. Paris : Frison-Roche, 24-26.

Borge, J. \& N. Viasnoff. 1995. Archives des métiers : les médecins. Milan : Éditions Michèle Trinckvel. Dictionnaire Français de Médecine et Biologie. 1970-75. 4 volumes. Paris : Masson.

Guérin, R. \& J. Thieulle. 1994. Lexique des termes médicaux et infirmiers et lexique étymologique. Paris : Lamarre.

Hamburger, J. 1982. Introduction du langage de la médecine., Paris : Flammarion Médecine-Sciences. Nouveau Larousse Médical. 1993. Paris : Larousse.

Vial, R. 1995. La chronologie de l'histoire de la médecine. Paris : Jean-Paul Gisserot.

\section{Relatives à la didactique}

Bérard, E. 1991. L'approche communicative. Collection DLE. Paris : Clé International.

Lehmann, D. 1993. Objectifs spécifiques en langue étrangère. Paris : Hachette.

Richterich, R. \& H.G. Widdowson. 1982. Description, présentation et enseignement des langues étrangères. Paris : Hatier.

Tagliante, C. 1994. La classe de langue. Paris : CLE International.

Widdowson, H.G. 1981. Une approche communicative de l'enseignement des langues. Collection LAL.

Paris : Hatier.

\section{NOTES}

1. Nous signalons que la description de l'attribution initiale de patronymes de savants aux référents n'est pas l'objectif de cette communication.

2. Il faut noter que ce chimiste étudia les poudres et les explosifs et inventa la dynamite.

3. Cette " gelée ", extraite d'une algue rouge, est notamment utilisée pour les milieux de culture en bactériologie 


\section{RÉSUMÉS}

Cet article sur les termes éponymes anglais ou français en médecine montre que cette sorte de dénomination n'est pas seulement caractérisée par certaines de ses difficultés sémantiques, mais aussi par les limites de la notion de termes éponymes, les traits dénotatifs et connotatifs de certains termes éponymes (en particulier, des connotations nationales, religieuses et culturelles). Il souligne la fréquence basse des termes éponymes employés par la communauté de spécialistes concernée. Il insiste aussi sur les traits historiques et humains de ces termes. Enfin, il présente dix exercices à partir de termes éponymes en médecine qui sont toujours absents, à notre connaissance, dans les manuels disponibles.

This paper on English or French eponymous terms in medicine shows that this kind of denomination is not only featured by some of its semantic difficulties but also by the limits of the notion of eponymous terms, the denotative and connotative characteristics of some eponymous terms, (specially cultural, religious and national connotations). It underlines the low frequency of eponymous terms used by the community of experts concerned. It also points out the historical and human features of these terms. Finally, it presents ten exercises based on eponymous terms in medicine, none of these types of exercises having yet been published, as far as we know, in existing textbooks.

\section{INDEX}

Mots-clés : connotation, dénomination, dénotation, difficulté sémantique, éponyme, exercice pédagogique, fréquence, médecine

Keywords : eponym, frequency, medicine, pedagogical skill, semantic difficulty

\section{AUTEUR}

\section{SYLVIE MONIN}

Sylvie Monin enseigne à l'Université Claude Bernard - Lyon 1. monin-badey@wanadoo.fr 